\title{
Torsten Wilholt
}

\section{Think about the Consequences! Nominalism and the Argument from the Philosophy of Logic}

\author{
[Manuscript version. The original publication appeared in
}

Dialectica 60 (2), 2006, 115-133.]

\section{Abstract}

Nominalism (the thesis that there are no abstract objects) faces the task of explaining away the ontological commitments of applied mathematical statements. This paper reviews an argument from the philosophy of logic that focuses on this task and which has been used as an objection to certain specific formulations of nominalism. The argument as it is developed in this paper aims to show that nominalism in general does not have the epistemological advantages its defendants claim it has. I distinguish between two strategies that are available to the nominalist: The Evaluation Programme, which tries to preserve the common truth-values of mathematical statements even if there are no mathematical objects, and Fictionalism, which denies that mathematical sentences have significant truth-values. It is argued that the tenability of both strategies depends on the nominalist's ability to account for the notion of consequence. This is a problem because the usual meta-logical explications of consequence do themselves quantify over mathematical entities. While nominalists of both varieties may try to appeal to a primitive notion of consequence, or, alternatively, to primitive notions of logical or structural possibilities, such measures are objectionable. Even if we are equipped with a notion of either consequence or possibility that is primitive in the relevant sense, it will not be strong enough to account for the consequence relation required in classical mathematics. These examinations are also useful in assessing the possible counter-intuitive appeal of the argument from the philosophy of logic.

\section{Introduction}

The nominalist's aim is to revamp ontology by denying the existence of abstract objects. The constructive part of the project is to explain how talk and reasoning about abstract entities, their non-existence notwithstanding, can still be helpful in our dealing with the world of concrete things. Some expressions that 
purportedly refer to abstract entities can reasonably be argued to be merely convenient paraphrases for more complicated assertions about concrete objects. They are consequently of little worry to the nominalist. But some abstract objects and their uses stubbornly resist an easy nominalistic explanation in this vein. The most notorious case is that of classical mathematics with its scientific (and everyday) applications to concrete phenomena.

Many philosophers have been motivated to try and tackle this problem for mainly epistemological reasons: Suppose, for the moment, that there were such things as numbers-how could we claim to know anything about them? How could our beliefs, that are in the end nothing but states of concrete organisms, be reliably correlated with facts about things that exist outside space and time and are assumed to be causally inactive and impassive? ${ }^{1}$ I think that the nominalists' worries have to be taken seriously and their quest to rid the world of this epistemological problem respected. Like many others, I think that they have so far not succeeded and that we should rather face this problem directly. How we might manage to do so is a different question, to which I have tried to contribute my share elsewhere (Wilholt 2004). In this paper, I will pursue two quite different aims.

The first aim is to survey a certain type of argument that intends to show nominalism's incapability of providing a solution to the aforementioned epistemological worries. This argument, which might be called 'the argument from the philosophy of logic', concentrates on the notion of consequence (and other related notions) employed in nominalistic theories. It shows how this notion, if regarded under the metaphysical restrictions of nominalism, incorporates all the epistemological problems that the nominalists wanted to get rid of in the first place. This argument has been put forward against specific varieties of nominalism and it has been taken to indicate a fundamental intricate entanglement of logic, modality and ontology (cf. especially Shapiro 1993, 1997: ch. 7; also Resnik 1983, 1985, Parsons 1990). My survey of the argument from the philosophy of logic in this paper is intended to provide a presentation of it that is independent of the technicalities of one or the other nominalistic project and to show that it can in one way or another be raised against any version of nominalism. It thus amounts to an objection against epistemological motivations for nominalism in general. Underlying this effort is a second, equally important aim. The argument from the philosophy of logic seems oddly counter-intuitive. (How can a consideration of the innocent notion of consequence have so much significance for our theory of what there is?) I

\footnotetext{
${ }^{1}$ See Field $(1989$, ch. $1, \S 4 \mathrm{~B}$ and ch. 7, §2) for a more detailed presentation of this reliabilist version of the access problem.
} 
will explore some of the intuitions involved in order to explain why the argument seems so counter-intuitive at times and why it is nonetheless valid. It should be emphasised that I will limit the discussion to matters directly pertinent to the argument from the philosophy of logic as I understand it, and that the paper must therefore not be mistaken for a survey of nominalism in general.

Epistemological concerns are not the only possible reason for being a nominalist. For example, a philosopher in pursuit of ontological parsimony per se might favour nominalism even when she is not interested in epistemology at all. The argument from the philosophy of logic has no force against this kind of nominalism. It works as an objection only against the widespread epistemological motivations of nominalism. To me, this makes it no less interesting, because these motivations offer a prima facie perspicuous reason why it might be better to omit certain objects from our ontology and to me this is what makes nominalism an important position to deal with. But it is a restriction of the argument from the philosophy of logic that must be acknowledged.

This restriction will also provide me with a defining constraint for the kind of nominalism that is treated in this paper. There are varying opinions concerning the question regarding what resources exactly the consistent nominalist is restricted to. Arguing against nominalism, I will be generous regarding this point. But I will insist that the nominalist must at least abstain from using resources that imply the same (or worse) problems of epistemic access as mathematical entities. Mathematical entities will thus serve as paradigmatic abstract objects. This has the advantage that though views concerning the exact meaning of the concept "abstract" may differ, numbers, sets, functions and their like all seem to fall safely within its extension.

I will leave out almost all the details of how the nominalist may explain the applicability of mathematics, and concentrate on only one aspect: How can we distinguish statements like

$$
2+2=4
$$

from statements like

$$
2+2=5
$$

if the entities they purport to refer to do not exist? I presuppose that some such distinction must underlie every successful nominalistic account of the usefulness of mathematical statements. Some nominalists may want to argue that sentences like $[\mathrm{P}]$ are in fact true, even though no such thing as the number 2 exists, and thus that the difference between sentences $[\mathrm{P}]$ and $[\mathrm{Q}]$ is just that 
one is true and the other false. The truth of $[\mathrm{P}]$ must then be explained in some non-standard way, and the nominalists in question must explain how. I will call this branch of nominalism the "Evaluation Programme", since it necessarily involves a truth-evaluation of all mathematical statements by non-referential means. ${ }^{2}$ Other nominalists will prefer to insist that $[\mathrm{P}]$ cannot be true when the singular terms " 2 ", etc. do not have referents. They will have to provide us with a non-standard account of how statements like $[\mathrm{P}]$ are distinguished from statements like [Q]. Following Hartry Field, I will label this second kind of nominalism "Fictionalism".

\section{An Introduction to the Evaluation Programme}

The Evaluation Programme is not overtly popular within current philosophy of mathematics. However, below I will point out a way to conceive of Geoffrey Hellman's Modal Structuralism as a very nuanced and sophisticated version of it. What is more, my personal experience is that belief in the Evaluation Programme is very widespread within the mathematical community. We should therefore take it seriously and try to find a convincing elaboration.

This elaboration must consist in an account for the truth of true mathematical statements (and for the falsity of the false ones) without reference to abstract entities. In general, the truth-values of mathematical sentences seem to be determined and fixed. ${ }^{3}$ The evaluation programme must identify the principle that is responsible for this determination. That is, it must specify a principle by which the values TRUE and FALSE are distributed over the totality of well-formed mathematical sentences.

In order to account for the empirical fact that doing mathematics is possible for finite beings, the principle of truth-value distribution sought by the Evaluation Programme should be some kind of rule (or set of rules) - for a rule is the only thing that could make this infinite assignation humanly manageable. It is only due to the fact that mathematics is axiomatisable that there actually is a candidate for a rule that could serve as an appropriate evaluation principle. The rule must be some variety of the principle: "For a mathematical sentence, to be true is to follow from a suitable set of axioms." I will assume that this principle can not be interpreted as "to be true is to be formally deducible from a

\footnotetext{
${ }^{2}$ The name is borrowed from Detlefsen (1986, esp. p. 33), who distinguishes two basic strategies for the explanation of the applicability of mathematics: a realist one, called "Interpretation", and an instrumentalist one called "Evaluation".

${ }^{3}$ Some may argue that there are exceptions to this rule (such as the continuum hypothesis). But this does not weaken the point here as long as there remains an infinite body of determined sentences when we have subtracted the exceptions.
} 
suitable set of axioms". The well-known problems of incompleteness would force us to abandon bivalence for mathematical statements if we adopted this simplistic formalism, and I take it for granted that most present day philosophers find this very unattractive. I therefore presuppose that the Evaluation Programme must rely on a semantic consequence relation.

According to the semantic conception, a sentence $X$ follows from a class of sentences $K$ if and only if $X$ is true under every such interpretation that makes all the elements of $K$ true. This conception thus quantifies over interpretations each of which involves a universe of discourse. Naturally, it is of great importance what the resource of objects for these universes of discourse looks like. Suppose, e.g., that this resource consisted of only 999999 different objects-then the sentence "Brutus is an honourable man" would follow logically from a statement to the effect that there are 1000000 different things (formulated by means of logical vocabulary). To avoid such absurdities, the object-resources for the interpretations must be as rich as possible. ${ }^{4}$

In contemporary logic, this requirement is met by agreeing on the settheoretical universe as the resource for interpretation. The all-purpose objects furnishing all kinds of universes of discourse for all kinds of interpretations are set-theoretical constructs, taken from the ontology of ZFC, our standard set theory. Obviously, the vertiginous ontology of ZFC constitutes a paradigm collection of just those abstract things whose existence the nominalist denies. At least for the time being, we must therefore treat the semantic consequence relation $\vDash$ as unattainable to the nominalist.

\section{A Common Sense Approach to Consequence}

The Evaluation Programme thus faces the difficult problem of explaining what "following from the axioms" could mean for a nominalist if our best theories of logical consequence presuppose an ontology of abstract objects. There is, however, a possibility to overcome this problem that has, to my mind, initially a very strong intuitive appeal. It involves an appeal to common sense. Don't we all, the proponent of the Evaluation Programme could say, have a very clear

\footnotetext{
${ }^{4}$ Interestingly, the problem would be much restricted if we confined ourselves to first-order logical consequence. It would suffice to make sure that the resource consists of (countably) infinitely many objects. For it would then follow from the downward Löwenheim-Skolem theorems that every first-order theory that has a model at all had a model composable from our object-resource. However, we will not limit our interest to first-order consequence and do not believe the sympathiser of the Evaluation Programme should do so, given her dependence on "suitable sets of axioms". Not even arithmetic can be formalised by a finite set of first-order axioms.
} 
idea of what it means for one sentence to follow from another? Logical consequence is a relation that, as a matter of fact, holds between some sentences. Obviously, we have the ability to grasp this relation. We have also devised theoretical tools to study this relation, like proof theory and model theory, that happen to presuppose abstract objects. But this does not force us to see the consequence relation itself contaminated by abstract ontology. How could nominalism, a very straightforward and fundamental thesis about what kinds of things there are, be doomed because contemporary formal logicians happen to insist on the ontology of set theory? In short: The advocate of the Evaluation Programme could claim that we do simply have an intuitively clear notion of logical consequence that is independent of metalogical theory and can be taken as primitive.

To say that some notion is primitive can mean one of (at least) three things, and it is important to be clear about in just what sense a concept is claimed or required to be primitive. (i) To claim that a notion is primitive may just mean that it will not be defined or explained in the theory at issue. (ii) Or it may mean that it refers to a basic element of the subject matter under discussion, in the sense of an element that is not composed of or constituted by any elements less complex than itself. (iii) Thirdly, it may mean that the application of the concept in question is epistemologically harmless, i.e. that we know whether or not it applies in every relevant case and it is possible to explain how we know this.

In our case, the purpose of the assumption that logical consequence is a primitive notion is to disperse the ontological worries that come with the semantic conception of consequence-that it contains an implicit quantification over the objects of ZFC. As my argument is restricted to epistemological motivations of nominalism, I will assume that the worries about quantification over the cumulative hierarchy stem from the concern that pure sets are not the kind of thing that we can plausibly know anything about. Therefore, in order to ultimately dispense with these worries, the claim must be that the notion of consequence is primitive in the third sense.

I regard the belief in an independent and immediately clear notion of consequence as an important factor in the nominalism issue, though it is hardly discussed in the literature (with some notable exceptions ${ }^{5}$ ). To assess its initial plausibility and thereafter probe its durability, consider the following quite plain example of a sentence following from two axioms.

\footnotetext{
${ }^{5}$ Discussions akin to what follows can be found in Rosemarie Rheinwald's excellent book (1984, ch. II) as well as in Putnam (1975, §3) and Parsons (1990, §§3-4).
} 
The "theory" generated by these two axioms is the familiar theory of halforder. It is a theory about only one dyadic relation, $<$. There are two axioms, $I$ and $T$, demanding irreflexivity and transitivity:

$$
\begin{gathered}
I \equiv_{\mathrm{df}} \quad \forall x \neg x<x . \\
T \equiv_{\mathrm{df}} \forall x \forall y \forall z \quad((x<y \wedge y<z) \rightarrow x<z) .
\end{gathered}
$$

A typical example for a half order is the proper subset relation, $\subsetneq$. Now consider the fact that the sentence $A$ (for "asymmetry") below follows logically from $\mathrm{I}$ and $\mathrm{T}$, regardless of what else than the axioms is true of " $<$ ".

$$
A \equiv_{\mathrm{df}} \forall x \forall y \neg(x<y \wedge y<x) .
$$

We can assure ourselves that it does so by means of a simple indirect consideration: Suppose that within an arbitrary half-order, there existed some $x$ and $y$ such that $x<y$ and at the same time $y<x$. Then, no matter what kind of relation $<$ is over and above being a half-order, it would have to follow from transitivity that $x<x$. But according to irreflexivity, this can never be the case with any half-order relation. The situation envisaged at the outset of our little deliberation can therefore never occur.

I agree that in this case, we can ascertain that any sentence of the form $A$ follows logically from sentences of the form $I$ and $T$ without having to invoke metalogic or any theoretically explicated concept of consequence. The example seems to show that the understanding that we can achieve in this way transcends the characteristics of specific relations like $\subsetneq$ and provides insight into the consequence relations between propositions that are due to their logical form. It is consequences of this kind that nourish the intuition that we possess a notion of consequence that is well understood and epistemologically harmless.

In the simple argument that we have used to arrive at this conclusion, we have implicitly quantified over binary relations on arbitrary totalities of objects, the characteristics of which were not further specified. We have simply reflected that if an arbitrary binary relation is irreflexive and transitive, then it is asymmetric.

In order to reconstruct this formally, let us express the statement that $I$ applies to an arbitrary binary relation $R$ on a totality of objects $M$ simply as the restriction of quantifiers in $I$ to $M$ and the replacement of every occurrence of < in $I$ by $R$. I will signify this result of restriction and replacement by $I_{R}^{M}$.

$$
I_{R}^{M} \equiv_{\mathrm{df}} \forall x(x \text { belongs to } M \rightarrow \neg R(x, x)) .
$$

$T_{R}^{M}$ and $A_{R}^{M}$ are to be formed analogously. With the aid of these expressions we can now give a truly simple and straightforward reconstruction of the consequence relation that we have in fact examined as follows: 
$[I+T \Rightarrow A] \quad \forall M \forall R(R$ is a binary relation on totality $M \rightarrow$ $\left.\left(\left(I_{R}^{M} \wedge T_{R}^{M}\right) \rightarrow A_{R}^{M}\right)\right)$.

It seems to be the case that there is an understanding of the consequence relation that makes it accessible to direct and pre-theoretical reflection foregoing metalogical investigations of structured sequences of symbol types (proofs), sets of sets (interpretations) or other sophisticated mathematicalia. I assume that the possibility of this straightforward understanding is exactly what fuels the intuition that we do possess an independent and immediately clear notion of consequence.

There might still be worries as to whether quantification over "totalities" and "relations" could possibly be available to the nominalist at all. However, I will assume that first-order totalities of concrete things and relations among these can themselves be understood as sufficiently concrete. I think this is plausible enough, considering that we are here concerned with a nominalistic reservation against abstract entities that rests on epistemological grounds. Since we have causal contact with collections, groups and aggregates of concrete things, an epistemology of totalities of concrete objects and their relations seems much less problematic than one for, say, pure sets. At any rate, I will grant so much to the nominalist that she can plausibly view some instances of the consequence relation as cases amenable to our straightforward understanding and thus graspable independently of any theory that includes commitment to abstract objects.

The hard problem for the straightforward understanding of consequence as envisaged in $[I+T \Rightarrow A]$ is a different one. The difficulty is simple (but devastating): Analyses like $[I+T \Rightarrow A]$ face the threat of being trivially satisfied. This would be the case if there were no totality in the whole universe that was half-ordered by any relation. For then, $I_{R}^{M} \wedge T_{R}^{M}$ would be false for all $M$ and $R$, such that $[I+T \Rightarrow A]$ would be trivially true. So likewise, it would be true that every arbitrary assertion whatsoever followed from $I$ and $T$ if understood analogously to $[I+T \Rightarrow A]$.

Of course, the threat is not factual in the special case of $[I+T \Rightarrow A]$ itself: We know many relations that constitute genuine half-orders among concrete objects. But simple examples for the intuitive clarity of the notion of consequence, like the present example of the theory of the half-order relation, are deceptive.

Contrast this with the example of "following from the axioms of arithmetic". Let $D$ be the conjunction of the Dedekind-Peano second order axioms of arithmetic. Analogously to our construction of $[I+T \Rightarrow A]$, we will understand under $D_{o, R}^{M}$ the result of restricting all quantifiers in $D$ to totality $M$ 
and replacing all occurrences of the successor relation in $D$ with $R$ and all occurrences of zero in $D$ with $o$. (If you like, you can read $D_{o, R}^{M}$ as " $M$ with $R$ as successor relation and $o$ as zero is an $\omega$-sequence.") Now consider the following attempt to capture the content of "Assertion $\phi$ follows from the axioms of arithmetic" analogously to $[I+T \Rightarrow A]$ :

$[D \Rightarrow \phi] \quad \forall M \forall R \forall o((R$ is a binary relation on totality $M \wedge o$ belongs to $M)$ $\left.\rightarrow\left(D_{o, R}^{M} \rightarrow \phi_{o, R}^{M}\right)\right)$.

$\left(\phi_{o, R}^{M}\right.$ stands for the result of the same replacements and restrictions in $\phi$ as described above for $D$.)

Now if there are no totalities $M$ at all possessing an element $o$ and a binary relation $R$ such that $D_{o, R}^{M}$, then $[D \Rightarrow \phi]$ would be trivially true for any arbitrary assertion $\phi$. In this case, it is far less than obvious that this will not happen to the nominalist, i.e. that there are in fact concrete totalities meeting the antecedent conditions (forming an $\omega$-sequence). What would such a totality consist of-the atoms of the universe? Space time points? The latter would probably be the safest bet-there are more than enough of them to save the day for $[D \Rightarrow \phi]$, provided they count as concrete objects.

However, I think it does not matter much whether we decide that space time points are concrete objects or not (cf. Field, 1989, ch. 6). The severity of the problem comes to the fore either way. For it is simply unacceptable to analyse the notion of consequence in such a way that whether or not some sentence follows from another depends on what kinds of totalities of concrete objects there are in the world. We are sure that it does not follow from the axioms of arithmetic that all even numbers are prime numbers, whether or not there exist infinite totalities of concrete entities that constitute $\omega$-sequences. We do not think that it would follow, even in a world where not a single such totality existed.

Coming from a realist, the foregoing argument requires comment. For it might seem that any realistic alternative will fare no better with regard to the dependence of logical consequence on what there is, and that the difference will simply be that a realist position will make the aforementioned arithmetical consequence dependent on whether or not there exist infinite totalities of abstract entities that constitute $\omega$-sequences. Is this not equally objectionable? The standard, realistically interpreted model-theoretic account of logical consequence, for instance, makes consequence dependent on the existence of sets and has in fact been rejected as an adequate explication for reasons related to this problem (cf. Etchemendy, 1990). But consider the following difference between the nominalistic and realistic situations. What seems intuitively 
objectionable about the nominalist's use of the straightforward analysis of consequence is that it makes logical consequence counterfactually dependent on what there is, or in other words, that it turns it into a contingent matter. In contrast, set-theoretical realists typically maintain that whatever sets there are exist necessarily, such that under the model-theoretic perspective, consequence is not a contingent matter. If the well-known oddities of ZFC have made you sceptical of set-theoretical realism, a more convincing realist alternative might be realism about structures. I think that for such a realism, a corresponding straightforward analysis of consequence is not a hopeless matter. ${ }^{6}$ If there are such things as abstract structures, they are arguably the same in every possible world, such that this perspective would not make logical consequence counterfactually dependent on what structures there are-not in the way that counterfactual dependence is usually understood. (Besides, even if one does not agree that whatever abstract structures there are exist necessarily, it may seem much less objectionable that whether or not one mathematical truth follows logically from another depends on what abstract structures exist, then that it depends on how many objects the universe contains.) These remarks do of course not establish a consistent realistic conception of consequence, which is not the purpose of this paper. They are only intended to show that the situations of realism and nominalism with respect to the aforementioned argument are not analogous.

For the purposes of nominalism, the straightforward analysis of consequence in the sense of $[D \Rightarrow \phi]$ is unhelpful, if it is understood as quantifying over concrete totalities. If I am right and this analysis is what we implicitly refer to when we rehearse considerations about the consequence relation like the one presented above for the case of $A$ following from $I \wedge T$, then it follows that our intuition that we have an immediate access to the notion of consequence (and can therefore treat it as primitive) is based on an illusion that may be caused by our preference for simple examples. In all cases where the premises of a consequence specify a structure that threatens to exceed the richness of concrete reality (as is easily the case with mathematical inferences), an implicit quantification over concrete totalities just will not do.

\section{Going Modal}

The nominalist may initiate a modal evasion manoeuvre. As a replacement for $[D \Rightarrow \phi]$ for example, she would then start from the following idea: In saying that

\footnotetext{
${ }^{6}$ I.e., a version of $[D \Rightarrow \phi]$ with $M$ ranging over abstract structures, $o$ over places in structures and $R$ over relations between places in structures.
} 
$\phi$ follows from $D$ we mean that, if there was a concrete totality constituting an $\omega$ sequence, then, without fail, $\phi$ would have to hold for it. A very sophisticated implementation of this idea can be found in Geoffrey Hellman's doctrine of Modal Structuralism (Hellman, 1989).

Hellman's programme is to provide a modal structural interpretation of mathematical theories that eliminates their reference to mathematical entities (ibid., 6). Even so, it may be fair to note that he does not call himself a nominalist and ultimately ends up expressing serious reservations about the nominalistic project (ibid., 116). However, the initial eliminative motivation makes it plausible to regard his doctrine as an improvement and elaboration of the Evaluation Programme and thus to include it in my assessment of nominalism's prospects (it should be noted that I am not the first one to do so, cf. Burgess and Rosen, 1997, sections II.C and III.A.I.c).

In response to the threat of trivial satisfaction, it would be comfortable to supplement $[D \Rightarrow \phi]$ with a categorical safeguard in the fashion of

$$
\exists M \exists R \exists o \quad D_{o, R}^{M} .
$$

But, as we have seen, for a nominalist this would be a precarious statement about the kinds of totalities that exist in the actual world. Mathematics cannot depend on what kind of structures there actually are (adopting Hellman's "structures" for our totalities-cum-relations). However, says Hellman, it depends on which structures are possible:

[M]athematics is the free exploration of structural possibilities, pursued by (more or less) rigorous deductive means. (Hellman 1989, 6)

Now, according to Hellman, we can provide a categorical safeguard for such explorations by means of a modalised statement. Whether or not any $\omega-$ sequence is actually realised, what matters is that it is possible that there was one. For Hellman, this must be a basic assumption of the science of arithmetic and takes the following form (ibid., 27):

$$
\exists M \exists R \exists o D_{o, R}^{M} .
$$

Of course, simplistic consequence notions like $[D \Rightarrow \phi]$ could still be trivially satisfied, given [C] (viz., if there does not exist an $\omega$-sequence in the actual 
world). Not so for Hellman's slightly more sophisticated analysis of "following from the axioms", which is likewise modalised: ${ }^{7}$

$$
\forall M \forall R \forall o\left(D_{o, R}^{M} \rightarrow \phi_{o, R}^{M}\right)
$$

Hellman understands these modalisations as providing a modal structural interpretation of $\phi$, consisting of one categorical [C] and one hypothetical component $[\mathrm{H}]$. In words that are not Hellman's own, his Modal Structuralism can be rakishly described as follows: When a mathematician states $\phi$, what she really, ultimately asserts is $[\mathrm{H}]$ and, as $[\mathrm{H}]$ 's backing, $[\mathrm{C}]$. This is how Hellman can declare $\phi$ to have a determinate truth value and explain this without invoking numbers: $\phi$ inherits the truth value of its modal structural interpretation, $[\mathrm{C}] \wedge[\mathrm{H}] ; \phi$ itself is not given an interpretation, and $[\mathrm{H}]$ and $[\mathrm{C}]$ quantify over other things, not numbers. In this vein, arithmetical sentences are provided with truth values without being interpreted; and this is why Hellman's eliminativism, if understood as a nominalistic endeavour, qualifies as a sophisticated version of the Evaluation Programme.

Naturally, the question arises how Hellman's modality is to be understood and, given that, how it fares epistemologically. Hellman treats the modal operator in question as a primitive (ibid., 8). Of course the modal notions should be primitive in sense (iii), which presupposes that their application poses no serious epistemological problems. More precisely, the epistemological problems, if any, of the modal notions should be distinctively less serious than the access problem for mathematical entities.

Stewart Shapiro sees in Hellman's strategy a fruitless attempt at a trade-off between ontology and modality, which cannot lead to a positive balance on the epistemological side (which he, too, presupposes to be the motivating concern behind nominalism/eliminativism): "Instead of an epistemology of abstract objects-numbers, sets, or structures-we now need an epistemology of possible abstract objects." (Shapiro, 1993, 466) While I endorse the general drift of this complaint, note that Hellman is even more radical than Shapiro assumes. He explicitly rejects "quantification over abstract structures, possible worlds, or intensions" and declares Barcan's formula not to hold for his modalities, so that the possible existence of a structure is not equivalent to the existence of a possible structure (Hellman, 1989, 16 f.). There can therefore allegedly be structural possibilities that do not involve ontological commitment to possible structures. I am not aware of any conclusive argument that would rule out this

\footnotetext{
${ }^{7}$ Cf. Hellman 1989, 23. To be sure, it is my imputation that [H] serves as Hellman's analysis of what it means to follow from the axioms. He himself only refers to it as the hypothetical component of $\phi$ 's modal structural interpretation.
} 
possibility, but it surely leads to pressing questions: How are we to investigate or even understand structural possibilities and their limits without reference to possible structures or possible worlds? Hellman's retreat from possibilia, though advisable to anyone with nominalistic intentions, has also moved him further away from our hard-earned customary understanding of modal notions. They are primitive, and we have to accept them or leave them, that is about all there is left to say about them.

\section{How Primitive is Modality?}

I have doubts about the implication, contained in Hellman's required sense of primitiveness, that the modal concepts are epistemologically unproblematic in their application. My claim is that this is not only unsatisfying (because it does nothing to improve our understanding), but that it is misleading. The central question is: Does the nominalist make any epistemic gain by going modal? Hellman's modalisations do not rest on a sophisticated epistemology of modality - and neither do those of Field or any other nominalist that I know of. Then what is their confidence in the epistemological lucrativeness of modalisation based on? My conjecture is that it is based on intuitions like the following:

[I] It is intuitively clear that it is easier to explain how we can know that an $\omega$ sequence is possible than to account for our knowledge of abstract, acausal natural numbers.

You may consider this a fourth, extended concept of primitiveness: (iv) A concept is primitive if its application is epistemologically harmless, i.e. if it is guaranteed and explainable that we know whether or not it applies in every relevant case, and if it is intuitively clear that it is guaranteed and explainable that we know whether or not it applies in every relevant case. This sense of primitiveness explains why some authors see no need for further epistemological explanations once a concept is declared primitive. If a workable epistemology of modality is absent, it is primitiveness in sense (iv) that structural possibilities need to possess.

I do not deny that this idea has a certain appeal-especially when one regards a claim like [I], which seems quite plausible to me. However, by considering different examples of possibility claims, this sense of intuitive clarity often evaporates quickly. Let us begin close to home. In everyday circumstances, we often use modal notions where it seems obvious that they are primitive in sense (iv):

[P1] Yes, it is possible to leave the university building via that staircase. 
Here, our knowledge of possibilities clearly derives from experience-personal or reported. We have been there, done that, therefore it must be possible. But possibility statements need not be that extremely concrete to appear perfectly harmless:

[P2] It is possible to correlate the Wonders of the Ancient World and the Deadly Sins one-to-one.

No one of our personal acquaintances has ever been there, no one may ever have done that, and still: No one would really object that the grasp of this possibility requires access to abstract entities like possible structures or possible worlds, or so the nominalist may argue.

I will not make a case that even everyday modal claims carry with them ontological commitments to possibilia or other abstract entities. In fact, I think that for homely examples like [P1], this would overshoot the mark. I also admit that it is plausible to extend this concession of intuitively clear epistemological innocence to some "structural" possibilities like [P2]. But this is only because in asserting [P2], though it is not directly based on experience like [P1], we are still extrapolating from experience. In other words: Our epistemic access to structural possibilities is unproblematic, and it is intuitively evident that this is so, as long as these possibilities are ultimately spatio-temporal possibilities, or more generally physical possibilities. I take it that we can arrive at physical possibilities by means of extrapolation from experience, in the sense that physically possible is whatever is not ruled out by the regularities that govern the physical world. We have, e.g., experience in collecting and correlating things, and we know from experience that there are few requirements for things to be correlated, except that they be distinct. Imagine for a moment a world where we could not have any such collecting and correlating experiences: [P2] would clearly not retain its appearance of intuitively evident epistemological innocence in that world.

Of course, we live in this world where we do seem to be able to accumulate all kinds of experiences with structures. So if these experiences suffice to sustain a primitive modal notion of "structural possibility", then what is the problem? The problem is that even in our world there are limitations to the kinds of structures we can gather experience about, and that these concrete structures will not suffice to provide the modal notions required for the complete body of classical mathematics.

A radical case in point is set theory. Consider the following line of reasoning, adapted from Charles Parsons (who presented it in a different argumentative context; Parsons, 1983). Concrete structures, whatever they are 
exactly, should in some sense be realised in space and time. We can understand this to imply that the concrete objects that constitute these structures should each be associated, however loosely or vaguely, with spacetime regions. To the best of our knowledge, the set of spacetime points has cardinality $2^{\aleph_{0}}$. The cardinality of the set of different spacetime regions, whatever gerrymandered or bizarre "regions" you may allow, can thus not exceed $2^{2^{\mathrm{x}_{0}}}$. Therefore, even if we allow that several concrete objects may inhabitate exactly the same spacetime regions (as long as "several" does not mean more than $2^{2^{\mathrm{x}_{0}}}$ again), the entirety of all concrete objects cannot surpass $2^{2^{\mathrm{x}_{0}}}$ in cardinality.

Mathematical set theory, in contrast, studies structures far beyond this limit. It requires, e.g., that there exist sets with cardinality $\beth \omega$, which is the smallest cardinality greater than all the cardinalities in the series

$$
\aleph_{0}, 2^{\aleph_{0}}, 2^{2^{\aleph_{0}}}, \ldots
$$

The possibility of structures with cardinality $\beth_{\omega}$ that mathematicians presuppose cannot implicitly indicate a physical possibility, nor can it be epistemically based on our experiences with concrete structural possibilities. As Parsons remarks:

Whatever convinces us, for example, that "there is" a cardinal number $\beth_{\omega}[\ldots]$ surely does not convince us that a structure of that cardinality having any but the most bloodless reality is possible: if concreteness demands causal efficacy, or perceivability in some strong sense, or temporality, or even some genuine individuality, we have no reason whatsoever to believe that $\beth_{\omega}$ can be concretely represented. (Parsons, 1983, 191 f.)

Our confidence that a set of cardinality $\beth_{\omega}$ is possible can thus not rest on our belief that it is realisable in concrete objects and their relations. Accordingly, the claim that our epistemological access to this possibility is intuitively clear seems suddenly exceedingly far-fetched. This is because the sense of intuitive clarity is limited to those modalities that can, at least in principle, be recovered in the world of experience. It is surely tempting to claim that our epistemic access to a structural possibility like [P2] is less difficult than our access to the number seven. But how would this claim be justified if [P2] was not so closely related to spatio-temporal possibilities as it obviously is? How could it be extended to cover the structural possibilities presupposed by the set-theoretic hierarchy, which are surely not spatio-temporal or even remotely physical?

I am not proposing that some sense of physical or nomological possibility represents the only meaningful notion of modality there is. The philosophical 
discussion has certainly exposed a variety of other promising modal notions. However, their standard semantic frameworks already presuppose objects (possible worlds, trans-world identity relations and so forth) whose epistemological status is certainly not preferable, from a nominalist perspective, to that of, say, numbers. So in any case, a modality presupposed by the nominalist needs an epistemological short-cut around possible worlds. Obvious candidates would be physical and spatio-temporal possibilities in the actual world, but, as the above considerations have shown, this will not do.

The perceived intuitive clarity of the epistemological innocence of structural possibilities is deceptive, I believe. The modality may therefore not be presupposed as primitive in sense (iv). But anyone who wanted to presuppose it as primitive in the weaker sense (iii) would have to supplement this declaration with an explicit justification of its epistemological innocence, presumably involving an epistemology of modal claims that fares without reference to abstract entities (at least without such abstract entities that are epistemologically as bad as numbers). ${ }^{8}$

Modalities thus lead to epistemological problems that are no easier than the ones that epistemologically motivated nominalists wanted to escape from in the first place. It would be inappropriate to protest against this conclusion by insisting that it has not been demonstrated that the modalities lead to problems as long as there might still be a satisfying epistemology of the relevant modal claims. For in this respect, nominalistic modalists are exactly on a par with their realist competitors: For all we know, there might be an epistemology for numbers, sets and the like that deals with the nominalists' worries. The gains expected from the nominalistic project are therefore not delivered; what we have instead is (at best) an epistemological tie. And in that case, it seems to be the most natural (and definitely the most naturalistic) choice to give preference to the hypothesis that preserves our everyday and scientific locutions by taking them at face value. Note again that all this critique does not affect Hellman's own well-balanced position, but only those who would try to employ his eliminative Modal Structuralism for nominalistic purposes. ${ }^{9}$

\footnotetext{
${ }^{8}$ My worry here is not just that nominalists have not provided one. The above considerations about the gap between physical possibilities and those structural possibilities presupposed in mathematics also cast into doubt the hope that an epistemology for the required modality can conceivably be provided on the basis of the purely concrete. But since I have no conclusive argument for this impossibility, I will insist only on the fact that it has not actually been achieved.

${ }^{9}$ Cf. Hellman 1989, 143 f., for his sober conclusions on the trade-off between the modal approach and platonism.
} 
The modalisation, I conclude, is not apt to elucidate the consequence relation from a nominalistic standpoint-not in a way that advances our epistemic situation. And the consequence relation would need some elucidation because its claim to intuitive perspicuity does not extend beyond the most undemanding examples due to the threat of trivial satisfiability. So long as these problems are not solved, it is hard to imagine how the Evaluation Programme is ever supposed to work out.

\section{Fictionalism}

An evaluation of the Evaluation Programme alone cannot amount to an adequate appraisal of nominalism's prospects of disposing of the epistemological problems. The Programme's problems seem to arise from its pretence to provide all mathematical sentences with non-trivial truth values. But the nominalist still has an alternative to this venture: Fictionalism. However, I believe that the same problems that I have already identified within the Evaluation Programme surface again for the fictionalist.

The view that mathematical statements do not really bear the truth-values we were once taught they bear is called Fictionalism, because it makes them resemble statements about fictional characters. "In Crete, there once lived a creature with a bull head and a human body" is to the best of our knowledge not a true statement, but it is licensed by Greek mythology, as opposed to, e.g. "The Minotaur was a duck in a sailor suit", which is neither true nor licensed in any way whatsoever. In the same way, fictionalists will have to explain how the practice of mathematics succeeds in licensing some statements, such as " $2+2=4$ ", and thus distinguishing them from others, like " $2+2=5$ ", even though both are likewise false.

As Stephen Yablo nicely puts it, Fictionalism in general turns mathematical entities into creatures of make-believe. Truth is not what matters about the statements concerning them, but rather validity within the fiction, or, in Yablo's terms, within the make-believe game (henceforth "validity", for short). From this point of view, the question is how a fiction or a game can flesh out such creatures sufficiently to determine, for any mathematical sentence, whether it is valid or not. For creatures of make-believe, as Yablo concedes,

... tend to have not much more to them than what flows from our conception of them. The green-eyed monster has no 'hidden substantial nature'; neither do the real-estate bug, the blue meanies, the chip on my shoulder, etc. (Yablo 2000, 225) 
But how do all the valid or invalid statements about mathematical entities "flow from our conception of them"? The fictionalist too will have to look for means to distribute a binary characteristic over the totality of well-formed mathematical sentences - only it's not the values TRUE and FALSE, but VALID and INVALID that must be distributed. And again, as in the case of the Evaluation Programme, the only candidate principle we have at our disposal to help make this infinite task manageable is the fact that mathematics is axiomatisable. For fictionalists, it can be put to service along the following lines: Our metamathematical investigations into the axiomatic structures of mathematics have shown us that the finite mathematical fictions manage to provide characterisations of the fictional entities they are about. So the statements licensed by the fiction are just those that follow from these characterisations. This is how the valid statements flow from our mathematical conceptions. The dependence of this general scheme on the notion of "following from characterisations" will bring with it problems analogous to those of the Evaluation Programme. What is at issue for the fictionalist purposes is the fullblown notion of consequence; and I have argued that we need mathematical theories to get a grip on this notion.

An analogous problem was first diagnosed for Hartry Field's sophisticated version of Fictionalism. In Field's view, the feature of mathematical statements that is relevant to their usefulness is not truth but conservativity (Field, 1980, ch.1). A statement counts as conservative if and only if we can add it to any nominalistic theory $N$ and still be certain that no nominalistic statement follows from $N+S$ that does not already follow from $N$ alone. (A nominalistic theory or sentence is one that does not include reference to or quantification over abstract entities.) Reviewers immediately criticised that his main argument for conservativeness of classical mathematics $S$ was a model-theoretic one ${ }^{10}$ thus effectively establishing a notion of conservativeness that implicitly contained quantification over set-theoretic constructs (cf. Malament, 1982, esp. 530, Resnik, 1983, esp. 517 f. and Detlefsen, 1986, 22 f.).

In response to this criticism, Field now maintains that the consequence relation in the definition of conservativeness is to be understood neither prooftheoretically nor model-theoretically, but in terms of a primitive notion of logical possibility and necessity, where $B$ being a consequence of $A$ means nothing but $\square(A \rightarrow B)$. According to Field, nominalists are nonetheless not left

\footnotetext{
${ }^{10} \mathrm{Ibid} ., 16-19$. He also provides a proof-theoretic argument, but this makes use of the completeness theorem and is thus restricted to first order languages, which are insufficient even for the nominalisation of Newtonian gravitation, as Field himself concedes: ibid., 38-40 and 115, note 30 .
} 
to their bare intuitions in evaluating logical possibility. They can make use of nominalistic versions of the usual metalogical devices. A sufficient criterion for logical possibility, e.g., is provided by a nominalistic version of the usual model-theoretic criterion:

$$
\square(T \rightarrow \text { there is a model of } A) \rightarrow \diamond A,
$$

where $T$ is the mathematical theory that governs model theory, i.e. a finitely axiomatised set theory (Field, 1989, 108-110 and 1991, 11-17). Since belief in [M] does not presuppose belief in $T$, so the argument goes, nominalists too are fully entitled to use it in their explorations of logical possibility. Again, the problems of the fictionalist mirror those of the Evaluation Programme. While [M] does not depend on $T$, it surely presupposes $\diamond T$, lest it be trivially satisfied. Now the nominalist is not to be blamed for not being able to provide a conclusive argument in favour of $\diamond T$ - for neither can anybody else. We all confidently hope that $\diamond T$. But from the nominalist perspective, it is hard to see how " $\diamond T$ " could even be understood. While for mathematical realists, it means that $T$ is realised or realisable in abstract structures, for nominalists, this way to conceive of it is simply another Platonist blunder. There are no abstract structures, so nothing whatsoever could be realised by them. And, as we have seen before, $T$ surely cannot be realised by concrete structures. In what sense can it nonetheless be comprehensible to assert that $T$ is logically possible?

Nominalistic versions of metalogical criteria, like $[\mathrm{M}]$, can therefore not help the nominalists to overcome the awkward epistemological situation that their appeal to primitive modal notions has created, because the trustworthiness of criterion [M] itself depends on a modal claim that is epistemologically problematic from a nominalistic point of view. This means that with Field, too, we are ultimately left with nothing but a modality we are supposed to accept as not being open to further explanation or analysis. ${ }^{11}$

\section{Conclusion}

In Field's case, as in the case of the Evaluation Programme, it is the notion of consequence (this time embedded within the conservativeness concept) that proves at the same time to be indispensable and deeply problematic for the epistemological motivation nominalists. Without it, the nominalist cannot explain how mathematical practice succeeds in providing us with the vast entirety of mathematical statements-no matter whether their distinguishing feature is thought to be (non-referential) truth, or conservativeness, or some

\footnotetext{
${ }^{11}$ For additional criticism of the unsolved epistemological problems of Field's modal concepts, see Resnik 1985, 169-175, and Shapiro 1997, 219-227.
} 
other kind of validity. But with it, she seems to be stuck with a notion that stands in need of explication, at least in so far as the full variety of consequence in classical mathematics is at issue, and that can only be satisfactorily explicated when totalities of abstract objects are included in our universe of discourse.

The argument from the philosophy of logic is thus a serious problem for the epistemological motivation of nominalism, as our survey of the argumentative situation shows. No matter whether the nominalist prefers the Evaluation Programme or Fictionalism à la Field and no matter whether she offers a modalised or non-modalised account of the story - the epistemological problems at issue can always be rediscovered, incorporated in one of the concepts presupposed by the respective account. Also, the counter-intuitive appearance of the argument from the philosophy of logic can be dispelled. The explanation for the perceived innocence of logical and modal notions is that we have some pre-theoretic grasp of such notions that is rooted in common sense and everyday experience. But there is no straightforward way to extend this pre-theoretic grasp to include the full range of our advanced mathematised reasoning about the world. The philosophy of logic and a basic question of ontology are thus connected in a perspicuous way. Without a sufficiently rich ontology, the notion of consequence is vacuous. What exactly "sufficiently rich" means depends on the varieties of consequence our logic is expected to cover. If it includes mathematical consequence, then the ontology that is in demand clearly exceeds anything the consistent epistemologically motivated nominalist can offer. The argument from the philosophy of logic should therefore be regarded as a coherent and serious counter-argument against epistemological motivations for nominalism.*

\section{References}

Burgess, J. P. and Rosen, G. 1997, A Subject with No Object: Strategies for Nominalistic Interpretation of Mathematics, Oxford: Clarendon

Detlefsen, M. 1986, Hilbert's Program: An Essay on Mathematical Instrumentalism, Dordrecht etc.: Reidel

Etchemendy, J. 1990, The Concept of Logical Consequence, Cambridge: Harvard University Press.

Field, H. 1980, Science Without Numbers, Princeton: Princeton University Press.

Field, H. 1989, Realism, Mathematics and Modality, Oxford: Blackwell

Field, H. 1991, "Metalogic and Modality”, Philosophical Studies 62, pp. 1-22

\footnotetext{
* I am grateful to Matthias Adam, Ansgar Beckermann and especially Christian Nimtz for their helpful remarks on an earlier version of this paper.
} 
Hellman, G. 1989, Mathematics Without Numbers, Oxford: Oxford University Press

Malament, D. 1982, Review of Field 1980, Journal of Philosophy 79, pp. 523-534

Parsons, C. 1983, "Quine on the Philosophy of Mathematics", in his Mathematics in Philosophy: Selected Essays, Ithaca: Cornell University Press, pp. 176-205

Parsons, C. 1990, “The Stucturalist View of Mathematical Objects”, Synthese 84, pp. 303-346

Putnam, H. 1975, "The Thesis that Mathematics is Logic", in his Mathematics, Matter and Method, (= Philosophical Papers, vol. 1), Cambridge etc.: Cambridge University Press, pp. 12-42

Resnik, M. D. 1983, Review of Field 1980, Noûs 17, pp. 514-519

Resnik, M. D. 1985, “How Nominalist Is Hartry Field's Nominalism?”, Philosophical Studies 47, pp. 163-181

Rheinwald, R. 1984, Der Formalismus und seine Grenzen: Untersuchungen zur neueren Philosophie der Mathematik, Königstein: Hain

Shapiro, S. 1993, "Modality and Ontology", Mind 102 (407), pp. 455-481

Shapiro, S. 1997, Philosophy of Mathematics: Structure and Ontology, Oxford \& New York: Oxford University Press

Wilholt, T. 2004, Zahl und Wirklichkeit: Eine philosophische Untersuchung über die Anwendbarkeit der Mathematik, Paderborn: Mentis

Yablo, S. 2000, "Apriority and Existence", in: P. Boghossian and C. Peacocke, eds., New Essays on the Apriori, London: Clarendon, pp. 198-228 